\title{
Métodos Temporais e Modelo de Deconvolução de Leray para as Equações de Navier-Stokes em Fluidos Incompressíveis via Elementos Finitos
}

\author{
Marcelo M. de Souza, Carolina C. Manica \\ Programa de Pós-Graduação em Matemática Aplicada, UFRGS \\ 91509-900, Porto Alegre, RS \\ E-mail: marcelo.maraschin@ufrgs.br
}

\begin{abstract}
Resumo: O estudo das equações de Navier-Stokes desperta interesse dos estudiosos da área da análise numérica, visto que a partir destas pode-se determinar os campos de velocidade e pressão de um escoamento. Com estas equaçôes também pode-se aproximar coeficientes aerodinâmicos, fato de grande interesse nas indústria automobilística e aeronáutica. Propõe-se estudar a aproximação das equações de Navier Stokes via o método de elementos finitos. Estudam-se duas propostas de métodos de discretização temporal para as equações dadas. Introduz-se um modelo de regularização e através do cálculo dos coeficientes de arrasto e sustentação comprova-se a sua efetividade.
\end{abstract}

Palavras-chave: Métodos Temporais, Modelo de Deconvolução de Leray, Elementos Finitos

\section{Introdução}

As equações de Navier-Stokes são equações diferenciais parciais que descrevem o escoamento de fluidos. A partir destas pode-se determinar os campos de velocidade e de pressão num escoamento. Logo, descrevem a física de um grande número de fenômenos de interesses econômicos e acadêmicos.

Estas equações são utilizadas para modelar o clima, correntes oceânicas, nos projetos de aeronaves e carros, nos estudos do fluxo sangüíneo, na análise dos efeitos da poluição hídrica em rios, mares e da dispersão da poluição atmosférica, etc.

Esse trabalho trata de fluido incompressível e viscosidade constante. Assim, as equações de Navier-Stokes bidimensionais incompressíveis em regime transiente, com condição de contorno de Dirichlet, são dadas por

$$
\begin{array}{rlll}
\mathbf{u}_{t}-\frac{1}{R e} \Delta \mathbf{u}+(\mathbf{u} \cdot \nabla) \mathbf{u}+\nabla p & =\mathbf{f} & \operatorname{em}(0, T] \times \Omega, \\
\nabla \cdot \mathbf{u} & =0 & \operatorname{em~}[0, T] \times \Omega, \\
\mathbf{u} & =0 & \operatorname{em~}[0, T] \times \partial \Omega, \\
\mathbf{u}(0, \cdot) & =\mathbf{u}_{0} & \text { em } \Omega,
\end{array}
$$

onde $\mathbf{u}=\left(u^{1}(x, y ; t), u^{2}(x, y ; t)\right)$ é a velocidade para duas dimensões, $p(x, y ; t)$ é a pressão, $\mathbf{f}=\left(f^{1}(x, y ; t), f^{2}(x, y ; t)\right)$ é a forçante, $R e$ é o número de Reynolds, $\Omega \subset \mathbb{R}^{2}$ é o domínio, $\partial \Omega$ é a fronteira, $\mathbf{u}_{0}$ é a velocidade inicial e $[0, T]$ é o intervalo de tempo.

Para obter uma formulação variacional para estas equações define-se o espaço da velocidade $X=H_{0}^{1}(\Omega)^{2}$ e da pressão $Q=L_{0}^{2}(\Omega)$, onde $H_{0}^{1}(\Omega)^{2}=\left\{\mathbf{v} \in L^{2}(\Omega)^{2}\right.$ e $\nabla \mathbf{v} \in L^{2}(\Omega)^{2 \times 2}: \mathbf{v}=$ 0 em $\partial \Omega\}$ é o espaço de Hilbert $\left(H^{1}(\Omega)^{2}\right)$ com condição de contorno zero e $L_{0}^{2}=\left\{q \in L^{2}(\Omega)\right.$ : $\left.\int_{\Omega} q d x d y=0\right\}$ é o espaço de Hilbert $H^{0}(\Omega)=L^{2}(\Omega)$ com média zero.

Para discretizar estas equações no espaço escolheu-se o método de elementos finitos, que tem se mostrado um ótimo método numérico na área de mecânica de fluidos. Uma introdução deste 
método aplicado a escoamentos viscosos de fluidos incompressíveis pode ser verificada em Layton [9].

Escolhe-se espaços de dimensão finita $X^{h} \subset X$ para a velocidade e $Q^{h} \subset Q$ para a pressão. Tome $X^{h}$ e $Q^{h}$ dados pelo elemento Taylor-Hood, ou seja, utiliza-se polinômios quadráticos na velocidade e lineares na pressão, veja mais em Layton [9]. A escolha por estes elementos se dá pelo fato de satisfazer a condição inf-sup discreta que garante admissibilidade dos dois espaços escolhidos para velocidade e pressão.

Assim, a solução aproximada por elementos finitos de (1)-(2) é: encontre $\mathbf{u}^{h}:[0, T] \rightarrow X^{h} \mathrm{e}$ $p^{h}:(0, T] \rightarrow Q^{h}$ satisfazendo

$$
\begin{aligned}
\left(\mathbf{u}_{t}^{h}, \mathbf{v}^{h}\right)+\frac{1}{R e}\left(\nabla \mathbf{u}^{h}, \nabla \mathbf{v}^{h}\right)+b^{*}\left(\mathbf{u}^{h}, \mathbf{u}^{h}, \mathbf{v}^{h}\right)-\left(p^{h}, \nabla \cdot \mathbf{v}^{h}\right) & =\left(\mathbf{f}, \mathbf{v}^{h}\right) \quad \forall \mathbf{v}^{h} \in X^{h}, \\
\left(\nabla \cdot \mathbf{u}^{h}, q^{h}\right) & =0 \quad \forall \quad \forall q^{h} \in Q^{h},
\end{aligned}
$$

onde $\mathbf{u}^{h}=\left(u^{h, 1}, u^{h, 2}\right)$ e (.,.) é o produto interno no espaço $L^{2}$.

As equações de Navier-Stokes consideradas neste trabalho estão em regime transiente, por isso o método de discretização temporal é muito importante no processo de obtenção da solução aproximada. Assim, um objetivo é comparar os métodos de discretização temporal.

\section{Métodos de Discretização Temporal}

Escolheu-se dois métodos de segunda ordem e implícitos. Segundo Schäfer e Turek [13] métodos explícitos podem ser ineficientes, pois, para satisfazer a condição de Courant-FriedrichsLewy necessitam de incremento temporal muito pequeno.

O primeiro é o método de Crank-Nicolson (CN), estudado para as equações de Navier-Stokes em Heywood e Rannacher [6], que além de ser de segunda ordem é A-estável. Crank-Nicolson é uma combinação dos métodos de Euler implícito e Euler explícito.

O outro método também de segunda ordem, desenvolvido em Bristeau et al. [2], é conhecido como método $\theta$ de passo fracionado (FS). Este método é fortemente A-estável e resolve o problema proposto em três etapas para cada passo de tempo.

Introduz-se $\theta_{i}$, para $i=1,2,3,4$, e $\Delta t_{k}$ o passo de tempo para o termo da pressão. Estes parâmetros são escolhidos de acordo com o método utilizado, segue na Tabela 1.

\begin{tabular}{|c|ccccccc|}
\hline Método & $\theta_{1}$ & $\theta_{2}$ & $\theta_{3}$ & $\theta_{4}$ & $t_{k}$ & $t_{k+1}$ & $\Delta t_{k}$ \\
\hline CN & 0.5 & 0.5 & 0.5 & 0.5 & $t_{n}$ & $t_{n+1}$ & $\Delta t$ \\
\hline FS & $\tau \theta$ & $\eta \theta$ & $\eta \theta$ & $\tau \theta$ & $t_{n}$ & $t_{n}+\theta \Delta t$ & $\theta \Delta t$ \\
& $\eta \theta^{*}$ & $\tau \theta^{*}$ & $\tau \theta^{*}$ & $\eta \theta^{*}$ & $t_{n}+\theta \Delta t$ & $t_{n+1}-\theta \Delta t$ & $\theta^{*} \Delta t$ \\
& $\tau \theta$ & $\eta \theta$ & $\eta \theta$ & $\tau \theta$ & $t_{n+1}-\theta \Delta t$ & $t_{n+1}$ & $\theta \Delta t$ \\
\hline
\end{tabular}

Tabela 1: Tabela de parâmetros para métodos temporais

Os coeficientes do método FS apresentados na Tabela 1 são escolhidos a fim de obter um esquema de segunda ordem e fortemente A-estável. Bristeau, Glowinski e Periaux [2] provam que deve-se escolher $\theta=1-\frac{\sqrt{2}}{2}, \tau=2-\sqrt{2}$ e $\theta^{*}=\eta=\sqrt{2}-1$.

Para obter a solução aproximada das equações de Navier-Stokes para fluidos incompressíveis discretizadas no espaço e no tempo, dado $\left(\mathbf{u}_{k}^{h}, p_{k}^{h}\right) \in\left(X^{h}, Q^{h}\right)$ encontre $\left(\mathbf{u}_{k+1}^{h}, p_{k+1}^{h}\right) \in\left(X^{h}, Q^{h}\right)$ satisfazendo

$$
\begin{aligned}
\left(\mathbf{u}_{k+1}^{h}, \mathbf{v}^{h}\right) & +\theta_{1} \Delta t\left[R e^{-1}\left(\nabla \mathbf{u}_{k+1}^{h}, \nabla \mathbf{v}^{h}\right)+b^{*}\left(\mathbf{u}_{k+1}^{h}, \mathbf{u}_{k+1}^{h}, \mathbf{v}^{h}\right)\right]-\Delta t_{k}\left(p_{k+1}^{h}, \nabla \cdot \mathbf{v}^{h}\right)= \\
& =\left(\mathbf{u}_{k}^{h}, \mathbf{v}^{h}\right)-\theta_{2} \Delta t\left[R e^{-1}\left(\nabla \mathbf{u}_{k}^{h}, \nabla \mathbf{v}^{h}\right)+b^{*}\left(\mathbf{u}_{k}^{h}, \mathbf{u}_{k}^{h}, \mathbf{v}^{h}\right)\right]+ \\
& +\theta_{3} \Delta t\left(\mathbf{f}_{k}, \mathbf{v}^{h}\right)+\theta_{4} \Delta t\left(\mathbf{f}_{k+1}, \mathbf{v}^{h}\right) \quad \forall \mathbf{v}^{h} \in X^{h}, \\
\left(\nabla \cdot \mathbf{u}_{k+1}^{h}, q^{h}\right) & =0 \quad \forall q^{h} \in Q^{h} .
\end{aligned}
$$


Uma dificuldade que se encontra nos métodos implícitos é a resolução de um sistema nãolinear em cada passo de tempo. Para isso existem métodos iterativos de linearização local, tais como Picard e Newton, veja em Gunzburger [4].

Um dos grandes problemas na obtenção da solução numérica da equações de Navier-Stokes em regime transiente é o alto custo computacional, devido a linearização do termo convectivo, da malha muito refinada e de passos de tempo pequenos. Por isso, busca-se alternativas que calculem as soluções encontradas com malhas finas em malhas grossas. Esta é uma das propostas do modelo de deconvolução de Leray, discutido em Layton et al. [10].

O modelo de deconvolução baseia-se na regularização introduzida por Leray [11], de um filtro diferencial discreto estudado por Manica e Kaya Merdan [12] e da aproximação de operadores de deconvolução de van Cittert, dado em [1].

\section{Modelo de Deconvolução de Leray}

Define-se o filtro diferencial discreto, dado em Manica e Kaya Merdan [12].

Definição 1:(Filtro diferencial discreto) Dado $\mathbf{u} \in L^{2}(\Omega)^{2}$, para um filtro de raio $\delta>0$, $\overline{\mathbf{u}}^{h}=A_{h}^{-1} \mathbf{u}$ é a única solução em $X^{h} \subset X$ de

$$
\delta^{2}\left(\nabla \overline{\mathbf{u}}^{h}, \nabla \mathbf{v}^{h}\right)+\left(\overline{\mathbf{u}}^{h}, \mathbf{v}^{h}\right)=\left(\mathbf{u}, \mathbf{v}^{h}\right) \quad \forall \mathbf{v}^{h} \in X^{h}
$$

Definição 2: Defina a projeção $L^{2}, \Pi^{h}: L^{2}(\Omega)^{2} \rightarrow X^{h}$ e o operador Laplaciano discreto $\Delta_{h}: X \rightarrow X^{h}$ por

$$
\left(\Delta_{h} \mathbf{u}, \mathbf{v}^{h}\right)=-\left(\nabla \mathbf{u}, \nabla \mathbf{v}^{h}\right),\left(\Pi^{h} \mathbf{u}-\mathbf{u}, \mathbf{v}^{h}\right)=0,
$$

para qualquer $\mathbf{v}^{h} \in X^{h}$.

Pode-se escrever que $A_{h}:=\left(-\delta^{2} \Delta_{h}+\Pi^{h}\right)$ e $\overline{\mathbf{u}}^{h}=\left(-\delta^{2} \Delta_{h}+\Pi^{h}\right)^{-1} \mathbf{u}$.

Considere o modelo de deconvolução de Leray:

$$
\begin{aligned}
\mathbf{u}_{t}+D_{N}(\overline{\mathbf{u}}) \cdot \nabla \mathbf{u}-\nu \Delta \mathbf{u}+\nabla p & =\mathbf{f} \quad \operatorname{em} \Omega \times(0, T), \\
\nabla \cdot \mathbf{u} & =0 \quad \mathrm{em} \Omega \times(0, T),
\end{aligned}
$$

onde $D_{N}$ é um operador de deconvolução de van Cittert, segue definição.

Definição 3: O operador de deconvolução discreto de van Cittert $D_{N}$ é definido por

$$
D_{N} \mathbf{u}^{h}=\sum_{n=0}^{N}\left(I-A_{h}^{-1}\right)^{n} \mathbf{u}^{h} .
$$

Por questões de notação, quando $N=0$ tome Leray0, quando $N=1$ tome Leray1, ou seja, para $N$ tem-se LerayN.

Segundo Dunca e Epshteyn [3] o operador de deconvolução $D_{N}$ satisfaz, para u suave,

$$
D_{N} \overline{\mathbf{u}}=\mathbf{u}+O\left(\delta^{2 N+2}\right) \quad N=0,1,2,3, \ldots
$$

Uma vantagem do modelo é que aumentando $N \rightarrow N+1$ aumenta a precisão de $O\left(\delta^{2 N+2}\right)$ para $O\left(\delta^{2 N+4}\right)$, mas isso requer a resolução de mais um problema de Poisson $\left(\left(-\delta^{2} \Delta+1\right)^{-1} \mathbf{u}\right)$ por $\Delta t$.

\section{Resultados}

Em ambos os exemplos desta seção o sistema linear é resolvido pelo método UMFPACK, que é uma versão sofisticada da fatoração LU. Utilizou-se o software livre FreeFEM ++ [5]. 


\subsection{Problema com solução exata conhecida}

Escolheu-se um problema de decaimento de vórtices. A solução exata de (1) - (2) é dada por

$$
\begin{aligned}
u_{1} & =-\cos (n \pi x) \sin (n \pi y) \exp \left(-2 n^{2} \pi^{2} t / \tau\right) \\
u_{2} & =\cos (n \pi y) \sin (n \pi x) \exp \left(-2 n^{2} \pi^{2} t / \tau\right) \\
p & =-0.25(\cos (2 n \pi x)+\cos (2 n \pi y)) \exp \left(-4 n^{2} \pi^{2} t / \tau\right)
\end{aligned}
$$

em um domínio $\Omega=(0,1)^{2}$. Considerou-se o termo de relaxação $\tau=1$ e a configuração de vórtices $n=4$. O lado direito $\mathbf{f}$, a condição inicial $\mathbf{u}_{\mathbf{0}}$ e as condições de contorno são obtidas a partir de que $\left(u_{1}, u_{2}, p\right)$ sejam soluções das equações de (1) a (4) para número de Reynolds igual a $1(\operatorname{Re}=1)$.

Utilizou-se malha regular com $h=1 / 64$. O tempo final deste escoamento é $T=1 s$ e o passo de tempo é dado por $\Delta t=0,1 \times 2^{-i}$, para $i=0, \ldots, 7$.

Para o termo não-linear utilizou-se a iteração de Picard em cada passo de tempo com critério de parada $\gamma<10^{-8}$, pois neste caso se mostrou mais rápido que o método de Newton, mas com resultados semelhantes.

Apesar de utilizar o elemento Taylor-Hood que satisfaz a condição inf-sup discreta, neste exemplo foi utilizado o método da penalidade para otimizar a pressão, veja mais em Gunzburger [4].

Na Figura 1 analisa-se os métodos temporais. Percebe-se que o erro na velocidade e na pressão é menor em praticamente todos os passos de tempo com o método FS do que com CN. Observa-se semelhança dos gráficos entre o presente trabalho e o artigo de John et al. [8].
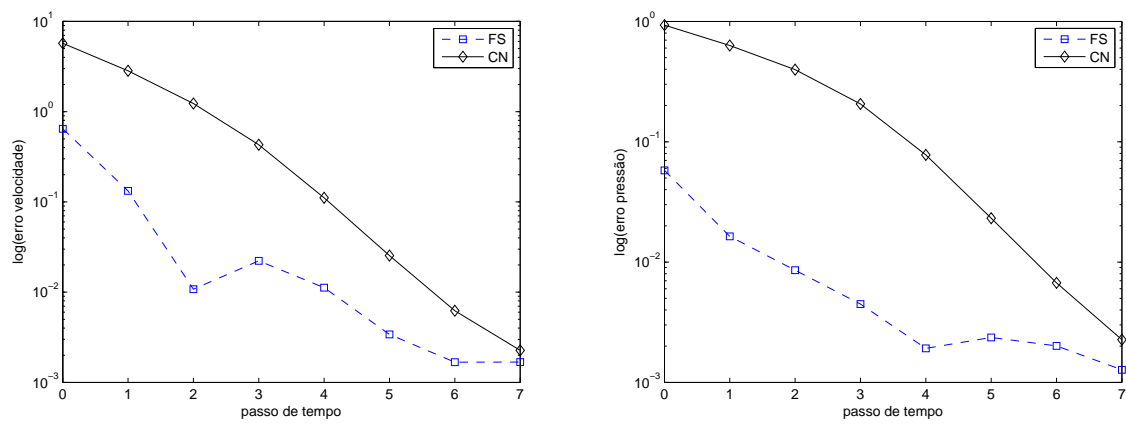

Figura 1: Cálculo dos erros da velocidade e pressão, respectivamente, para diferentes métodos de discretização temporal por passo de tempo

Outros exemplos com soluções exatas conhecidas podem ser encontrados em Souza [14].

\subsection{Simulação de Coeficientes Aerodinâmicos}

Trata-se do escoamento em duas dimensões em torno de um cilindro, onde a solução exata é desconhecida. Utilizou-se as equações de (1) a (4) com tais condições de contorno, $\mathbf{u}(t ; 0, y)=$ $\mathbf{u}(t ; 2.2, y)=0.41^{-2} \sin (\pi t / 8)(6 y(0.41-y), 0) \mathrm{m} / \mathrm{s}$ e condição de não-deslizamento nas partes superior, inferior e na superfície do cilindro. Considere $\nu=10^{-3} \mathrm{~m}^{2} / \mathrm{s}, \mathbf{f}=\mathbf{0}$ e tempo $T=8 \mathrm{~s}$.

Para o termo não-linear utilizou-se o método de Newton com critério de parada $\gamma<10^{-10}$, que mostrou-se mais eficiente que o método de Picard nesse caso.

Esse tipo de problema serve para testar a fidelidade física do algoritmo. Os coeficientes aerodinâmicos são números adimensionais utilizados para o estudo aeronáutico, aerodinâmico e automobilístico. Medem as forças que sofre um corpo qualquer em movimento num fluido. Alguns dos coeficientes mais conhecidos são o coeficiente de sustentação $\left(c_{l}\right)$ e o coeficiente de arrasto $\left(c_{d}\right)$. Outro parâmetro a ser estudado é a diferença de pressão entre parte da frente e de trás do cilindro dada por $\Delta p=p(8 ; 0.15,0.2)-p(8 ; 0.25,0.2)$ no tempo $T=8 s$. 
Para as computações foram escolhidos 3 níveis de malha. A malha 2 do presente trabalho tem a mesma quantidade de graus de liberdade da malha 1 do trabalho de John [7] e a malha 3 deste trabalho com a malha 2 de John [7]. Para o passo de tempo escolheu-se $\Delta t=0,02 / 2^{i}$, onde $i=0, \ldots, 4$.

Em Schäfer e Turek [13], este mesmo exemplo é resolvido, com diversos métodos de solução numérica e malhas bem refinadas. Conclui-se que o intervalo ótimo do coeficiente de arrasto é $2,93<c_{d, \max }<2,97$, o coeficiente de sustentação é $0,47<c_{l, \max }<0$, 49 e para a diferença da pressão o intervalo é $-0,115<\Delta p(8 s)<-0,105$. A Tabela 2 e a Tabela 3 podem ser comparadas com John [7].

\begin{tabular}{|c|c|c|c|c|c|c|}
\hline Nível & $\Delta t$ & $t\left(c_{d, \max }\right)$ & $c_{d, \max }$ & $t\left(c_{l, \max }\right)$ & $c_{l, \max }$ & $\Delta p(8 s)$ \\
\hline 2 & 0.02 & 3.96 & 2.966817 & 5.76 & 0.4252636 & -0.10341796 \\
\hline 2 & 0.01 & 3.94 & 2.9677771 & 5.72 & 0.4395816 & -0.10987897 \\
\hline 2 & 0.005 & 3.94 & 2.9682405 & 5.715 & 0.44286571 & -0.11106215 \\
\hline 2 & 0.0025 & 3.9375 & 2.9684635 & 5.7075 & 0.44233924 & -0.11141863 \\
\hline 2 & 0.00125 & 3.93625 & 2.968574 & 5.70875 & 0.44227988 & -0.11156644 \\
\hline 3 & 0.02 & 3.94 & 2.94787 & 5.76 & 0.454906 & -0.105271 \\
\hline 3 & 0.01 & 3.94 & 2.94886 & 5.72 & 0.468869 & -0.110712 \\
\hline 3 & 0.005 & 3.94 & 2.94933 & 5.705 & 0.470592 & -0.11146 \\
\hline 3 & 0.0025 & 3.9375 & 2.94956 & 5.7025 & 0.470288 & -0.111705 \\
\hline 3 & 0.00125 & 3.93625 & 2.94966 & 5.7025 & 0.470129 & -0.111824 \\
\hline
\end{tabular}

Tabela 2: Método Crank-Nicolson com simulação direta (DNS)

\begin{tabular}{|c|c|c|c|c|c|c|}
\hline Nível & $\Delta t$ & $t\left(c_{d, \max }\right)$ & $c_{d, \max }$ & $t\left(c_{l, \max }\right)$ & $c_{l, \max }$ & $\Delta p(8 s)$ \\
\hline 2 & 0.02 & 3.94 & 2.9670828 & 5.72 & 0.46536749 & -0.11087478 \\
\hline 2 & 0.01 & 3.94 & 2.9678846 & 5.71 & 0.45473204 & -0.1113912 \\
\hline 2 & 0.005 & 3.935 & 2.9682856 & 5.71 & 0.44797908 & -0.11156249 \\
\hline 2 & 0.0025 & 3.935 & 2.9684852 & 5.7075 & 0.44485994 & -0.11163467 \\
\hline 2 & 0.00125 & 3.935 & 2.9685848 & 5.70875 & 0.44322709 & -0.11166792 \\
\hline 3 & 0.02 & 3.94 & 2.94816 & 5.7 & 0.490578 & -0.111436 \\
\hline 3 & 0.01 & 3.94 & 2.94897 & 5.7 & 0.481648 & -0.111718 \\
\hline 3 & 0.005 & 3.935 & 2.94938 & 5.7 & 0.475536 & -0.111831 \\
\hline 3 & 0.0025 & 3.935 & 2.94958 & 5.7025 & 0.472414 & -0.111888 \\
\hline 3 & 0.00125 & 3.935 & 2.94968 & 5.70125 & 0.470737 & -0.111918 \\
\hline
\end{tabular}

Tabela 3: Método $\theta$ de passo fracionado com DNS

Fazendo uma comparação com o artigo de John [7] e com Schäfer e Turek [13] nota-se que os métodos CN e FS mostraram-se apropriados para o cálculo de todos os coeficientes. O coeficiente de sustentação é muito sensível e sofre bastante variação ao longo do tempo, por isso no nível 2 ainda não foi possível encontrar valores dentro do intervalo de referência de Schäfer e Turek [13], mas os valores encontrados neste trabalho estão mais próximos do intervalo ótimo do que em John [7].

Agora, serão feitas algumas análises deste exemplo utilizando o modelo de deconvolução de Leray. A grande vantagem do modelo estudado é obter as soluções esperadas com menos graus de liberdade que os exemplos com DNS.

Com uma malha no nível 1, conseguiu-se aproximar o coeficiente de arrasto a partir do modelo de deconvolução Leray1 e testou-se também para o modelo de deconvolução com Leray0 (ver Tabela 4). Considerou-se $\delta=\frac{\pi r}{n}$, onde $r$ é o raio do cilindro e $n$ é o número de pontos na borda do cilindro. Esta escolha de $\delta$ tem relação com o incremento da malha próximo ao cilindro, onde ocorre maior variação no escoamento.

Observe que somente com Leray1 são obtidos valores no intervalo de referência. Isso confirma a observação feita em Layton et al. [10], que ao longo do tempo os modelos de deconvolução de 
Leray com $\mathrm{N} \geq 1$ tendem a ficar corretos, enquanto com o modelo de Leray0, da mesma forma que com DNS, o erro pode crescer ao longo do tempo.

\begin{tabular}{|c|c|c|c|c|c|c|c|}
\hline- & - & \multicolumn{2}{|c|}{ DNS } & \multicolumn{2}{c|}{ Modelo Leray0 } & \multicolumn{2}{c|}{ Modelo Leray1 } \\
\hline Nível & $\Delta t$ & $t\left(c_{d, \max }\right)$ & $c_{d, \max }$ & $t\left(c_{d, \max }\right)$ & $c_{d, \max }$ & $t\left(c_{d, \max }\right)$ & $c_{d, \max }$ \\
\hline 1 & 0.02 & 3.92 & 2,8722666 & 3,92 & 3.107692 & 3,94 & 2,9615456 \\
\hline 1 & 0.01 & 3.92 & 2,8730993 & 3,93 & 3.1085435 & 3,92 & 2,9624477 \\
\hline 1 & 0.005 & 3.92 & 2,8735152 & 3,925 & 3.1089547 & 3,92 & 2,9628647 \\
\hline 1 & 0.0025 & 3,9175 & 2,8737253 & 3,925 & 3.109161 & 3,92 & 2,9630732 \\
\hline 1 & 0.00125 & 3.9175 & 2,8738297 & 3.92625 & 3.1092645 & 3,92125 & 2,9631777 \\
\hline
\end{tabular}

Tabela 4: Coeficiente de arrasto na malha 1 com FS

Com a malha no nível 2, conseguiu-se aproximar o coeficiente de sustentação no intervalo de referência a partir do modelo de deconvolução Leray3 (ver Tabela 5). Ainda $\operatorname{com} \delta=\frac{\pi r}{n}$.

A escolha por Leray3 é justificada pelo fato que Leray1 e Leray2 não aproximaram todos os coeficientes de maneira ótima. A partir disso confirma-se o argumento exposto anteriormente, que quanto mais filtros são aplicados, maior a ordem de aproximação do modelo de Leray.

Todas as simulações dos coeficientes estão no intervalo de referência de Schäfer e Turek. Note que o $c_{l}$ com DNS não era obtido no intervalo ótimo (ver Tabela 3 ).

\begin{tabular}{|c|c|c|c|c|c|c|}
\hline Nível & $\Delta t$ & $t\left(c_{d, \max }\right)$ & $c_{d, \max }$ & $t\left(c_{l, \max }\right)$ & $c_{l, \max }$ & $\Delta p(8 s)$ \\
\hline 2 & 0.02 & 3.94 & 2.968728 & 5.72 & 0.4912897 & -0.11062063 \\
\hline 2 & 0.01 & 3.94 & 2.9690268 & 5.71 & 0.48043859 & -0.11122779 \\
\hline 2 & 0.005 & 3.935 & 2.9694297 & 5.715 & 0.47249932 & -0.11142283 \\
\hline 2 & 0.0025 & 3.9375 & 2.9696298 & 5.7125 & 0.47194154 & -0.11150124 \\
\hline 2 & 0.00125 & 3.9325 & 2.9697301 & 5.7125 & 0.47083318 & -0.11153619 \\
\hline
\end{tabular}

Tabela 5: Modelo de Leray3 com método temporal FS e malha no nível 2

Por fim, na Figura 2, o gráfico com o histórico temporal do coeficiente de sustentação comparando os modelos com DNS nos níveis de malha 2 e 3 , e com o modelo de Leray3 com a malha no nível 2. Conclui-se que o modelo Leray3 aproximou o coeficiente de sustentação com um nível a menos de malha.

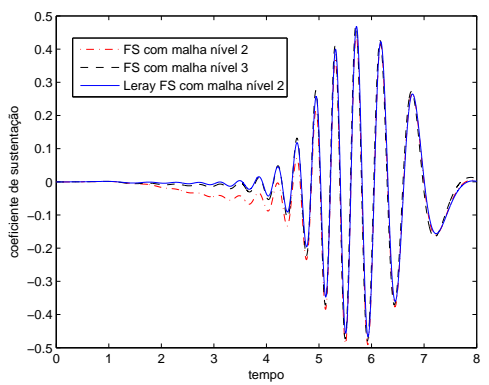

(a) Coeficiente de sustentação

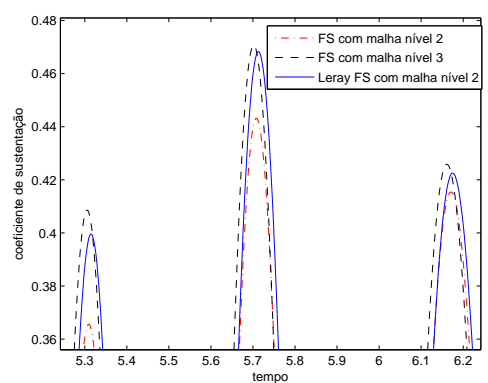

(b) Zoom ponto máximo

Figura 2: Evolução temporal do $c_{l}$ com o método temporal FS e $\Delta t=0.00125$

\section{Conclusão}

Os dois métodos temporais testados mostraram algumas diferenças. O método de $\mathrm{CN}$ aproximou bem as soluções das equações de Navier-Stokes, mas as soluções ficaram menos satisfatórias 
que com o método FS. Sabe-se que o método ser apenas A-estável faz com que tenha oscilações numéricas que podem ser corrigidas apenas com passos de tempo suficientemente pequenos, Turek [15]. Enquanto FS, por ser fortemente A-estável, é capaz de aproximar as soluções com passo de tempo maior sem apresentar instabilidade.

No exemplo onde simulou-se os coeficientes aerodinâmicos, observou-se que os resultados obtidos foram mais satisfatórios que em John [7], e ainda melhor se comparados com os resultados obtidos com a regularização de Leray, ver Tabela 5. Além disso, confirmou-se o fato do modelo de deconvolução de Leray aproximar as soluções em níveis de malha mais grossa, onde modelos com DNS não conseguem capturar tais soluções satisfatoriamente.

\section{Referências}

[1] M. Bertero and P. Boccacci. "Introduction to Inverse Problems in Imaging". Institute of Physics Pub., 1998.

[2] M. O. Bristeau, R. Glowinski, and J. Periaux. Numerical methods for the Navier-Stokes equations. applications to the simulation of compressible and incompressible viscous flows. Computer Physics Reports 6, pages 73-187, 1987.

[3] A. Dunca and Y. Epshteyn. On the stolz-adams deconvolution model for the large-eddy simulation of turbulent flows. SIAM Journal on Mathematical Analysis, 37(6):1890 - 1902, 2006.

[4] M. Gunzburger. "Finite Element Methods for Viscous Incompressible Flows: a Guide to Theory, Practice, and Algorithms". Computer science and scientific computing. Academic Press, 1989.

[5] F. Hecht. "Freefem ++ ". Laboratoire Jacques-Loius Lions, Université Pierre et Marie Curie, Paris, Third Edition, Version 3.19.

[6] J. Heywood and R. Rannacher. Finite-element approximation of the nonstationary Navier-Stokes problem. part iv: Error analysis for second-order time discretization. SIAM Journal on Numerical Analysis, 27(2):353-384, 1990.

[7] V. John. Reference values for drag and lift of a two-dimensional time-dependent flow around a cylinder. International Journal for Numerical Methods in Fluids, 44(7):777-788, 2004.

[8] V. John, G. Matthies, and J. Rang. A comparison of time-discretization/linearization approaches for the incompressible Navier-Stokes equations. Computer Methods in Applied Mechanics and Engineering, 195(44-47):5995 - 6010, 2006.

[9] W. Layton. "Introduction to the Numerical Analysis of Incompressible Viscous Flows". Computational Science and Engineering Series. Society for Industrial and Applied Mathematics, 2008.

[10] W. Layton, C. C. Manica, M. Neda, and L. G. Rebholz. Numerical analysis and computational testing of a high accuracy leray-deconvolution model of turbulence. Numerical Methods for Partial Differential Equations, 24(2):555-582, 2008.

[11] J. Leray. Essai sur les mouvements plans d'une liquide visqueux que limitent des parois. Journal de Mathématiques Pures et Appliquées, Paris, Série IX, 13:331-418, 1934.

[12] C. Manica and S. Kaya Merdan. "Convergence analysis of the finite element method for a fundamental model in turbulence". Technical report, University of Pittsburgh, 2006.

[13] M. Schäfer and S. Turek. Benchmark computations of laminar flow around a cylinder. Flow Simulation with High-Performance Computers II, 1996.

[14] M. M. Souza. "Solução das Equações de Navier-Stokes para Fuidos Incompressíveis via Elementos Finitos". Dissertação de Mestrado, PPGMAp-UFRGS, 2013.

[15] S. Turek. A comparative study of time-stepping techniques for the incompressible Navier-Stokes equations: From fully implicit non-linear schemes to semi-implicit projection methods. International Journal for Numerical Methods in Fluids, 22(10):987-1011, 1996. 